\title{
Valorisation de déchets de poisson alliés à des rejets brassicoles en vue d'obtenir un biofertilisant
}

\author{
TAIEK Taha', BOUTALEB Nadia',2, BAHLAOUAN Bouchaib',3, EL JAAFARI Amal ', KHROUZ Hssein', SAFI Amal' et EL ANTRI Said \\ 1. Laboratoire de Biochimie, Environnement \& Agroalimentaire URAC 36, Faculté des Sciences et Techniques, Université Hassan II Mohammedia-Casablanca \\ 2. Ecole National Supérieure d'Arts et Métiers de Casablanca \\ 3. Institut Supérieur des Professions Infirmières et des Techniques de Santé de Casablanca \\ 4. Société des Brasseries du Maroc, bd Ahl Loghlam, Sidi Moumen - Ain Sebaa - 20450 Casablanca
}

\section{Auteur/s à qui la correspondance devrait être adressée : boutalebensam@gmail.com}

\section{RESUME}

L'industrie agroalimentaire au Maroc génère continuellement de nombreux déchets organiques qui posent à la fois un problème économique et écologique. Leur valorisation peut pourtant donner naissance à une nouvelle source économique.

Le présent travail consiste à valoriser les déchets industriels de poissons en les combinant avec les biomasses de levure rejetées par le secteur brassicole, afin de créer un mélange de départ nutritivement équilibré comme fertilisant, de par sa contenance en élément de base : le triplet azote/carbone/phosphore.

Plusieurs mélanges binaires sont alors élaborés dans l'objectif de chercher la formule optimale. Les compositions ainsi préparées étaient suivies pendant quinze jours, à température ambiante et sous agitation continue en utilisant des agitateurs de paillasse. Le pH, la température et la conductivité étaient contrôlés chaque jour. D'autres contrôles, tels que la mesure de la matière sèche, l'azote, le phosphore, la triméthylamine ainsi que des tests microbiologiques, ont été menés en début, au milieu et à la fin de cette étude.

Les résultats montrent que le produit généré est mature au bout de huit jours, il est sans odeur, son pH est stable, présentant de bonnes qualités hygiéniques et il est riche en azote et phosphore. Les tests réalisés sur des espèces d'orge Hordeum vulgare et de tomates Lycopersicon lycopersicum, montrent qu'il permet d'améliorer les propriétés de croissance de leurs cultures.

MOTS-CLÉS : Déchets de poisson, levure de bière, biofertilisant, gestion des déchets.

\begin{abstract}
Food industry in Morocco generates continuously many organic wastes that pose both economic and environmental problem. Yet their valuation can procreate new economical source.

This study seeks to enhance industrial fish waste in combination with biomass yeast rejected by the brewing industry; to create a starting mixture of nutritionally balanced its contents in basic element: the triplet nitrogen / carbon / phosphorus.

Several binary mixtures are then developed with the aim of seeking the optimal formula. The compositions were followed for two weeks at room temperature under a continuous stirring using benchtop stirrer, $\mathrm{pH}$, conductivity and temperature were monitored daily. Other parameters, such as the measurement of the dry matter, nitrogen, phosphorus, trimethylamine and microbiological were controled in the beginning, middle and end of the study. The results show that product is mature after eight days, it is odorless, $\mathrm{pH}$ is stable, have good hygienic quality and it is rich in nitrogen and phosphorus. The tests on species of barley Hordeum vulgare and tomatoes Lycopersicon lycopersicum, show that it improves the growth properties of their cultures.
\end{abstract}

KEYWORDS : Fish waste, brewer's yeast, biofertilizer, waste management 


\section{Valorisation de déchets de poisson alliés à des rejets brassicoles en vue d'obtenir un biofertilisant}

\section{INTRODUCTION}

Au Maroc, le secteur de la pêche occupe une place importante dans l'économie nationale. Son développement est lié aux questions environnementales, notamment du point de vue de la gestion des déchets vues les quantités énormes générées. En effet, les quantités des déchets de poisson et de poissons non usinables et non transformés sont estimées à plusieurs centaines de milliers de tonnes de déchets par an (Chabbar, 1996 ; Afilal et al. 2014). Faute de moyens et/ou par absence de législations contraignantes ou enfin par lacune dans leur application, les mesures de traitement sont peu développées. Les déchets sont souvent déposés anarchiquement dans les décharges mal ou non contrôlées, avec des conséquences négatives sur la santé de la population limitrophe (El Moutawakil, 1995 ; Benzakour et al. , 2003).

Au cours de ces dernières années, la Société des Brasseries du Maroc a connu également une grande expansion dans le royaume. Elle figure parmi les industries considérées comme polluantes, ses rejets se caractérisant par une demande chimique en oxygène (DCO) très élevée qui varie entre I 50.000 à 250.000 mg/l (Leclercq et al. , 1985).

Comme le séchage n'est pas accessible à toutes les unités de production (du point de vue coût d'installation et besoins énergétiques), les procédés de biotransformation pour ces déchets semblent être les mieux appropriés pour pallier ces problèmes. L'application d'un procédé spécifique approprié aboutirait en effet à l'élaboration d'un produit bénéfique, d'origine naturelle, utilisable comme fertilisant pour les sols.

Le présent travail a pour objectif la valorisation des déchets de poissons grâce à un procédé simple et peu coûteux. Les biomasses de levure rejetées par le secteur brassicole sont nutritivement très riches. Leur exploitation dans le traitement des déchets de poissons en tant qu'agent de biotransformation et source de carbone serait d'un grand intérêt (Arneborg et al. , 1997).

TABLEAU I. Composition et propriétés initiales des essais

\begin{tabular}{|c|c|c|c|c|c|c|c|c|}
\hline Composition & $\begin{array}{l}\text { Déchets de } \\
\text { poisson (\%) }\end{array}$ & $\begin{array}{c}\text { Déchets de levure } \\
\text { brassicole (\%) }\end{array}$ & $\mathrm{pH}$ & $\begin{array}{l}\text { Tempéra- } \\
\text { ture }\left({ }^{\circ} \mathrm{C}\right)\end{array}$ & $\begin{array}{c}\text { Conductivité } \\
(\mathrm{mS} / \mathrm{m})\end{array}$ & MS \% & $\begin{array}{l}\text { Phosphore } \\
\text { (mg/lo0g) }\end{array}$ & $\begin{array}{l}\text { Azote } \\
\text { (g/loog) }\end{array}$ \\
\hline $\mathrm{Cl}$ & 100 & 0 & 6,54 & 18 & 1,74 & 53 & 28,3 & 0,83 \\
\hline $\mathrm{C} 2$ & 75 & 25 & 4,66 & $|8|$, & 3,45 & 36 & 21,1 & 1,14 \\
\hline C3 & 50 & 50 & 4,81 & 18 & 3,5 & 30,5 & 52,4 & 1,53 \\
\hline C4 & 25 & 75 & 4,85 & 17,3 & 3,53 & 28,6 & 48,75 & 1,2 \\
\hline
\end{tabular}


Quant au taux de phosphore, il est déterminé par dosage spectrophotométrique selon la Norme française NFVI 8- 106 (Afnor, 1980).

\section{I.3.Analyses microbiologiques}

L'analyse microbiologique est réalisée au premier, septième et dernier jour de la biotransformation. Un milieu Columbia au sang est préparé ; il permet de déterminer la présence spécifique de streptocoques à effets protéolytiques (Leyral et al. 2007). La présence de staphylocoques (marqueur lipolytique) est identifiable sur une gélose Chapman (Branger et al. , 2007) Un milieu Mac Conkey est utilisé pour visualiser la présence d'Escherichia coli comme indicateur d'hygiène (Zahar et al., 2002).

\section{I.4. Test de toxicité et de fertilisation}

L'étude de la toxicité du biofertilisant sur la germination des graines est réalisée sur l'espèce d'orge Hordeum vulgare (Amira) et l'espèce de tomate Lycopersicon lycopersicum (Campbell 33), originaires du Maroc (fournies par l'Institut National de la Recherche Agronomique). L'orge et la tomate sont cultivées dans des étuis d'une profondeur de $6 \mathrm{~cm}$ et les graines sont déposées respectivement à une profondeur de $3 \mathrm{~cm}$ et $5 \mathrm{~mm}$ puis recouvertes de terre. Le sol est mélangé à une fraction de $0,009 \mathrm{~g}$ par $200 \mathrm{~g}\left(6 \mathrm{~cm}^{2}\right.$ de sol) équivalent à I $50 \mathrm{Kg} /$ hectare (dose minimale recommandée par le FAO concernant l'utilisation des engrais pour les cultures céréalières au Maroc) (FAO, 2006). Chaque test a été reproduit 4 fois.

Deux plateaux de culture sont utilisés : un pour la culture d'orge et l'autre pour celle des tomates. Chaque plateau contient 6 colonnes et chaque colonne contient 4 étuis.

Les 8 étuis de la colonne 1 et 2 contiennent le sol témoin avec aucun rajout de fertilisant. Les 4 étuis de la colonne 3 contiennent le fertilisant commercial. Les étuis de la colonne 4,5 et 6 contiennent respectivement les essais C2, C3, C4 élaborés durant la présente étude.

\section{Résultats et interprétations}

\section{I. Aspects des mélanges}

Au début tous les essais formaient une pate épaisse très hétérogène. Au cours de la biotransformation une liquéfaction est observée d'une manière ascendante selon l'ordre CI, C2, C4 et C3. Une odeur de pourriture s'est développée dans $\mathrm{Cl}$ alors que C2, C3, C4 présentaient des odeurs peu importantes. Il y a eu une présence de vers au bout de 5 jours pour $\mathrm{Cl}$.

\subsection{Température}

La variabilité de la température représentée (figure I) est peu significative (variabilité maximale de $5^{\circ} \mathrm{C}$ ). L'évolution de la température permet de suivre l'évolution d'un tel processus et de distinguer entre les différentes phases qui succèdent.
Normalement, pendant la première phase, la chaleur produite par les micro-organismes impliqués dans la dégradation aérobie des sucres, les acides aminés libres et l'amidon devrait faire augmenter la température. Selon les quantités de matière impliquées, celle-ci est d'autant plus facilement évacuée lorsque l'épaisseur des essais manipulés est faible. L'agitation régulière appliquée durant le procédé permet en plus une bonne maitrise de l'élévation de la température

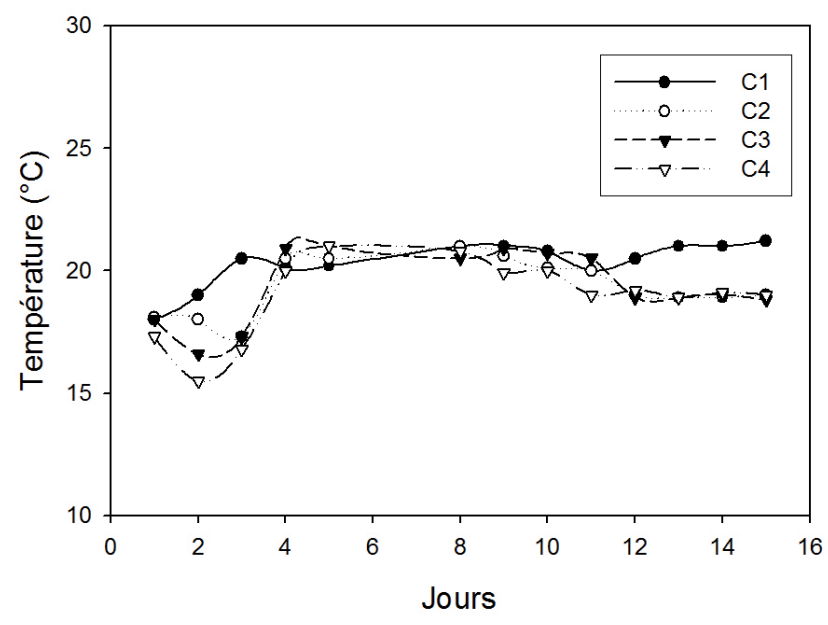

Figure I. Evolution de la température

\subsection{PH. Conductivité et matière sèche}

D'après les résultats de suivi du $\mathrm{pH}$ pendant 15 jours présentés dans la figure 2 , on observe que le $\mathrm{pH}$ initial des différents essais $\mathrm{C2}, \mathrm{C} 3$ et $\mathrm{C} 4$, est acide $(\mathrm{pH}=4,5)$, ce qui peut être dû à l'acidité de la levure brassicole ajoutée.

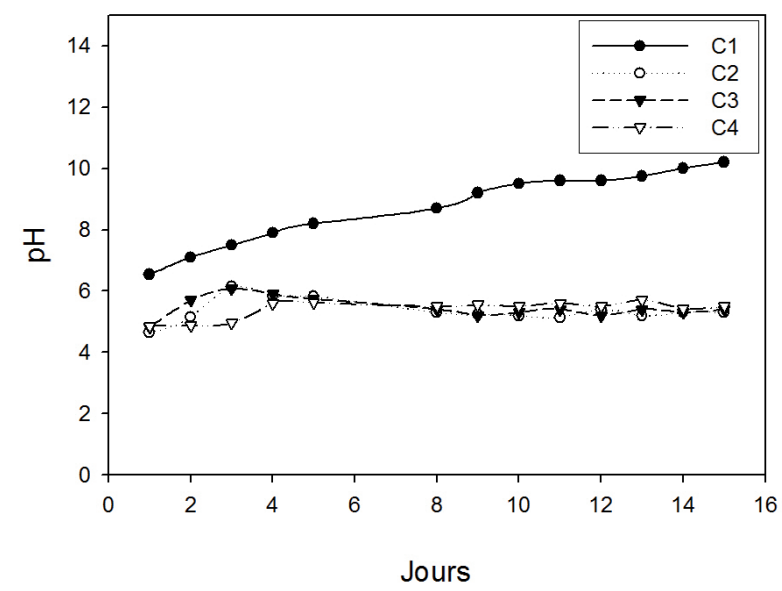

Figure 2. Evolution du pH

Comparés au témoin $\mathrm{Cl}$, les autres essais ont subi une légère augmentation de $\mathrm{pH}$ dans les 3 premiers jours. Le $\mathrm{pH}$ ne tarde pas à rejoindre la valeur initiale de 4,5 à partir du 4ème jour et reste stable le long de l'expérience.

Pour $\mathrm{Cl}$ le $\mathrm{pH}$ augmente progressivement jusqu'à la valeur de 10 au $15^{\text {ème jour. }}$ 


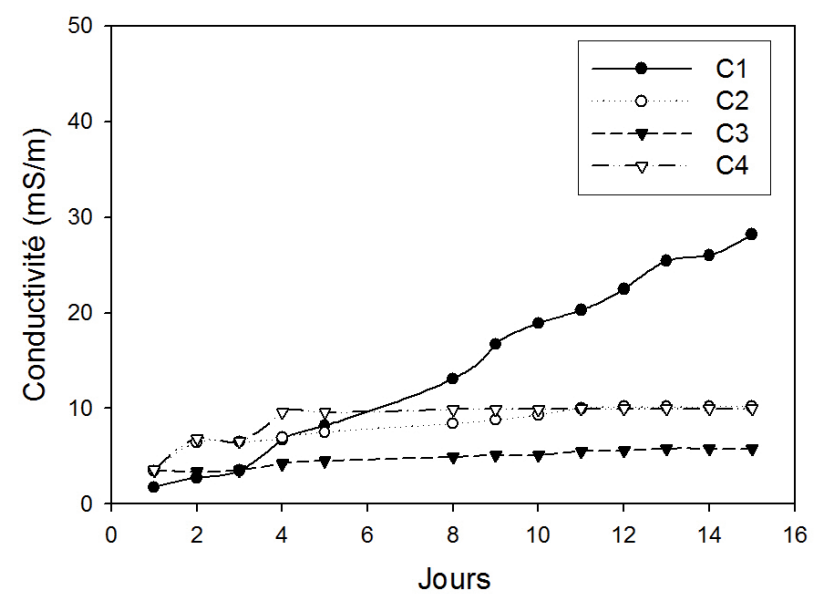

Figure 3. Evolution de la conductivité

D'après la figure 3, on remarque que la conductivité reste stable pour l'ensemble des essais à l'exception du témoin $\mathrm{Cl}$, où elle augmente remarquablement pour passer de 3 à 28 $\mathrm{mS} / \mathrm{m}$ après 15 jours.

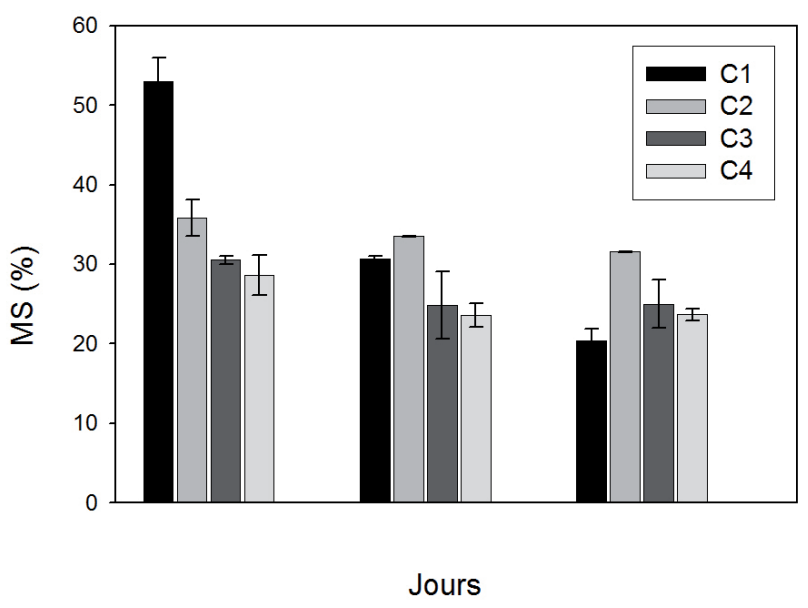

Figure 4. Evolution du taux de matière sèche (MS)

En parallèle avec les résultats précédents, la matière sèche des essais (Figure 4) diminue légèrement pour $C 2, C 3$ et $C 4$, contrairement à $\mathrm{Cl}$ où elle diminue remarquablement.

II est à noter que le contrôle de la matière sèche donne une idée sur combien, en matière, est perdue après qu'elle soit convertie en substance volatile suite à un processus de biotransformation. Les résultats de la figure 4 montrent le rôle que joue le rajout de la levure brassicole à orienter favorablement la biotransformation et à défavoriser éventuellement les réactions qui tendent à libérer une partie de la matière par conversion en substances volatiles (e.g. carbone organique sous forme de $\mathrm{CO}_{2}$, azote sous forme de $\mathrm{N}_{2}$ ).

\subsection{Phosphore,Azote total et Triméthylamine}

Les figures $5 \mathrm{~A}$ et $\mathrm{B}$ présentent l'évolution du taux de phosphore ainsi que l'azote total.

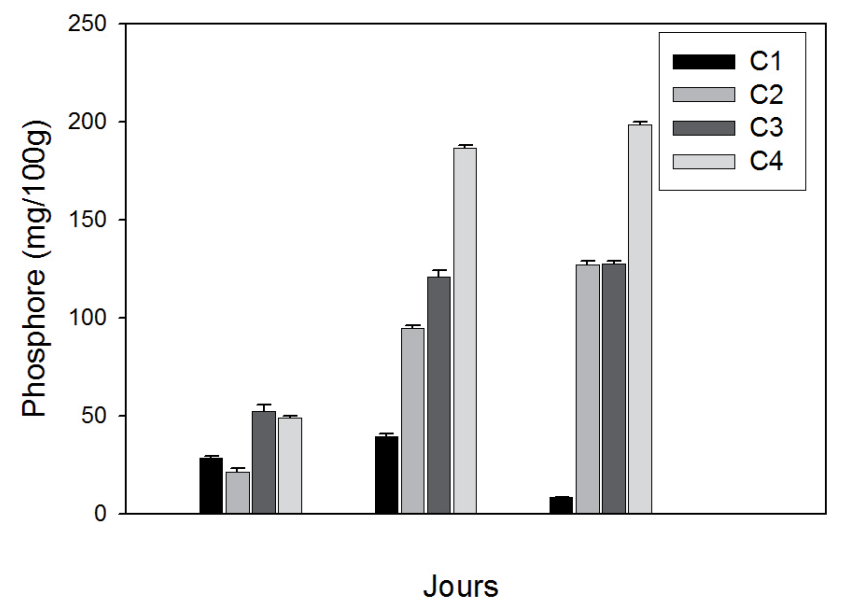

Figure 5. Evolution du taux de phosphore (A)

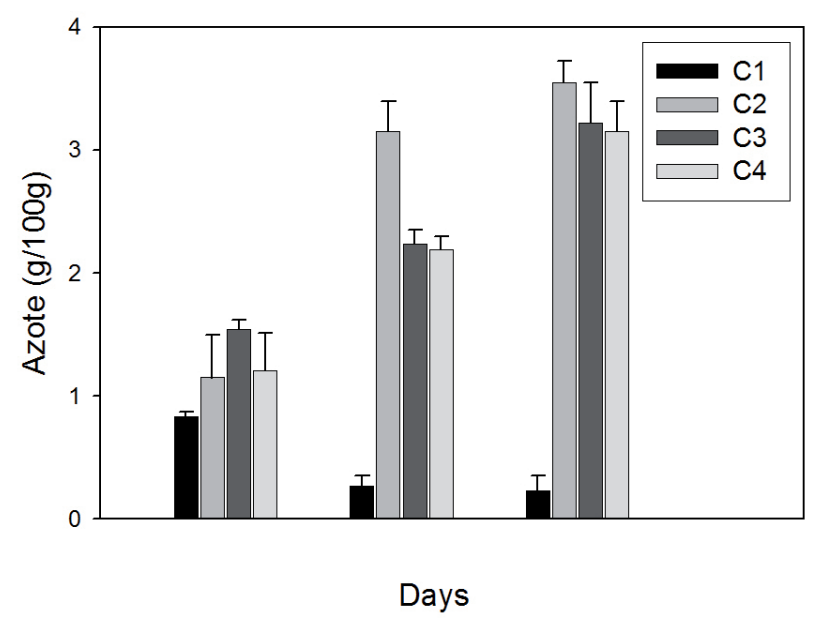

Figure 5. Evolution du taux d'azote (B)

On remarque que le taux de phosphore ainsi que l'azote marquent une baisse considérable pour le témoin Cl. Contrairement aux autres essais, ces taux ont augmenté durant le processus de fermentation.

Quant au taux de la triméthylamine il marque une légère diminution nettement exprimée dès le 8ème jour pour les essais C2, C3 et C4 (Figure 6). Par contre, il a subi une élévation considérable pour $\mathrm{Cl}$.

L'ensemble des résultats de contrôle physico-chimiques confirme l'effet favorable de la levure brassicole sur l'optimisation de la biotransformation des déchets de poissons.

Les paramètres de contrôle classique température, $\mathrm{pH}$ et conductivité montrent qu'après le huitième jour le processus de la biotransformation commence à se ralentir et se stabiliser. Le tableau 2 récapitule l'évolution parallèle des autres paramètres de contrôle, pour les différentes compositions, et ce au huitième jour de la biotransformation. 


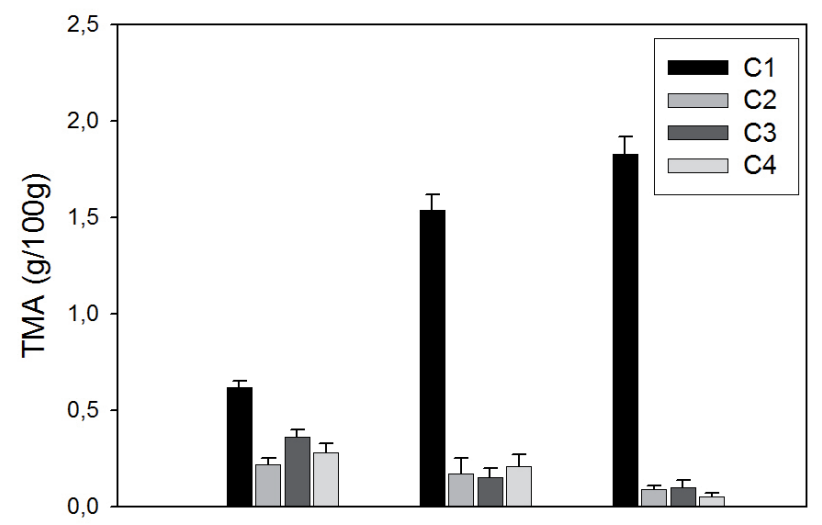

Days

Figure 6. Evolution du taux de la TMA dans les différents essais

Tableau 2. Evolution des paramètres physicochimiques au huitième jour de la biotransformation

\begin{tabular}{|c|c|c|c|c|}
\hline $\begin{array}{c}\text { Matière } \\
\text { sèche }\end{array}$ & $-42 \%$ & $\mathbf{- 7} \%$ & $-18 \%$ & $-18 \%$ \\
\hline $\begin{array}{c}\text { Phos- } \\
\text { phore }\end{array}$ & $+38 \%$ & $\mathbf{+ 3 4 9 \%}$ & $+131 \%$ & $+283 \%$ \\
\hline Azote & $-67 \%$ & $\mathbf{+ 1 7 4 \%}$ & $+45 \%$ & $+81 \%$ \\
\hline TMA & $+148 \%$ & $-\mathbf{2 3} \%$ & $-58 \%$ & $-25 \%$ \\
\hline
\end{tabular}

Note: +

Augmentation par rapport à l'origine -: réduction par rapport à l'origine

\section{Tableau 3. Analyse microbiologique après dilution au $1 / 10$}

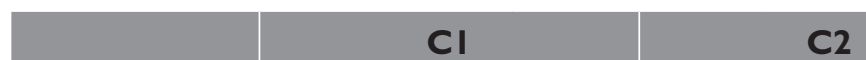

\begin{tabular}{|c|c|c|c|c|c|c|c|c|c|c|c|c|}
\hline \multirow[b]{2}{*}{ Jours } & \multicolumn{3}{|c|}{ CI } & \multicolumn{3}{|c|}{$\mathrm{C} 2$} & \multicolumn{3}{|c|}{ C3 } & \multicolumn{3}{|c|}{ C4 } \\
\hline & $0 \mathbf{j}$ & $\mathbf{7 j}$ & I5j & $0 \mathbf{j}$ & $\mathbf{7 j}$ & I5j & $0 \mathrm{j}$ & $7 \mathbf{j}$ & I5j & $0 \mathrm{j}$ & $7 \mathbf{j}$ & I5j \\
\hline Escherichia.coli & ++ & ++ & ++ & ++ & ++ & - & ++ & ++ & - & ++ & ++ & - \\
\hline Staphylocoques & + & + & + & + & + & - & + & + & - & + & + & - \\
\hline Streptocoques & + & + & + & + & + & - & + & + & - & + & + & - \\
\hline
\end{tabular}

Note: ++ :> 100 UFC; + :> 10 UFC; - :Absence

Tableau 4. Résultat du test de fertilisation sur la culture d'orge et de la tomate (2I jours, 4 répétitions).

\begin{tabular}{|c|c|c|c|c|c|c|c|c|}
\hline & \multicolumn{4}{|c|}{ Orge } & \multicolumn{4}{|c|}{ Tomate } \\
\hline & Germination & $\begin{array}{c}\mathrm{L}_{\mathrm{m}} \\
\text { tiges } \\
(\mathrm{mm})\end{array}$ & $\mathrm{L}_{\mathrm{m}}$ & $\begin{array}{c}\text { PS } \\
\text { plante (g) }\end{array}$ & Germination & $\begin{array}{c}L_{m} \\
\text { tiges }(\mathrm{mm})\end{array}$ & $\begin{array}{c}L_{m} \\
\operatorname{racines}(\mathrm{mm})\end{array}$ & $\begin{array}{c}\text { PS } \\
\text { plante (g) }\end{array}$ \\
\hline $\begin{array}{l}\text { Engrais } \\
\text { commercial }\end{array}$ & + & 15 & 13 & 1,01 & + & 8 & 9 & 0,74 \\
\hline$C 2$ & + & 18,5 & 28 & 1,9 & + & 10 & 14 & 1,2 \\
\hline $\mathrm{C} 3$ & + & 18 & 24 & ।,5 & + & 10 & 11 & I \\
\hline C4 & + & 18 & 23 & 1,6 & + & 9 & 11 & 0,9 \\
\hline
\end{tabular}

Note: + : Positive - : négative, Lm: longueur moyenne ; PS : poids sec 


\section{Discussion}

La baisse du pH dans les essais C2, C3 et C4 est due à l'activité fermentative des levures brassicoles Saccharomyces cerevisiae (Meyer et al. , 2004). Le suivi du pH indique qu'à partir du 8ème jour les essais $\mathrm{C} 2, \mathrm{C} 3$ et $\mathrm{C} 4$ sont matures. Le pH est stabilisé suite à la réduction de l'activité des micro-organismes (Albrecht, 2007). Pour le témoin Cl qui ne contient que les déchets de poisson seul, il s'alcalinise en fonction du temps, ce qui indique que sa transformation est différente du reste des essais. Globalement l'évolution de la conductivité est corrélée au $\mathrm{pH}$ : plus le $\mathrm{pH}$ s'éloigne de la neutralité plus la conductivité est élevée, ce qui est dû vraisemblablement à l'augmentation de la présence des formes ioniques.

Avant les 4 premiers jours l'évolution de la conductivité est deux fois plus importante pour les essais C2, C3 et C4 par rapport à $\mathrm{Cl}$ en raison de leurs richesses en sels minéraux qu'apporte la levure brassicole (MC Cance, 2002 ; Patrie, 2008). Celle-ci finit par se stabiliser à 10 mS/m après ce terme. Tandis que pour $\mathrm{Cl}$ la valeur de la conductivité continue à augmenter après le 4ème jour pour atteindre environ 28,2 $\mathrm{mS} / \mathrm{m}$ au I 5 ème jour suite à une grande liquéfaction et dégradation de la matière. II est évident que la présence des ions pourrait être bénéfique pour les plantes mais il a été démontré qu'une conductivité trop élevée menace la survie des microorganismes et réduit alors la qualité des engrais (Sherstha et al. , 200I).

La matière sèche $(M S / M)$ finale est comprise entre 20 et 31,5\% pour tous les essais dans l'ordre croissant $\mathrm{Cl}>\mathrm{C} 4>$ C3> C2 conformément à leurs aspects de liquéfaction. Les pertes par rapport aux MS initiales au I 5 ème jour varient alors de $62 \%$ pour Cl à $12 \%$ pour C2. La diminution est due à la dégradation des glucides par les levures et au dégagement des substances volatiles (Lourhzal et al. , 2008).

Concernant le taux du phosphore initial, il est compris entre 28 et $52,5 \mathrm{mg} / \mathrm{l00} \mathrm{g}$ pour tous les essais dans l'ordre croissant $\mathrm{C} 2>\mathrm{Cl}>\mathrm{C} 4>\mathrm{C} 3$ du fait qu'ils contiennent de la levure brassicole avec différents pourcentages riche en sels minéraux et en phosphore (Patrie, 2008). Tous ces essais ont un taux de phosphore qui augmente pendant les 8 premiers jours. Cela est dû à la minéralisation réalisée par les micro-organismes qui transforment le phosphore protéique en phosphore minéral (Albrecht, 2007). Au I5ème jour l'augmentation est plus importante pour l'essai C2 et C4, respectivement d'environ $502 \%$ et $307 \%$. L'ajout de la levure brassicole aux mélanges favorise donc la conversion du phosphore.

Au cours de la fermentation, la teneur en azote total augmente respectivement au I 5 ème jour de 1 I0, 162, 208\% pour les essais C3, C4, C2. Contrairement aux autres compositions, $\mathrm{Cl}$ exprime une diminution en azote total de $75 \%$. Ceci est expliqué par l'activité des micro-organismes et la perte de la fraction volatile et liquide.

Concernant la triméthylamine elle est comprise entre 0,05 et 0,1\% pour les essais dans l'ordre croissant C4> C2>C3 au I 5 ème jour : cette diminution significative prouve la maîtrise du procédé de fermentation. Par contre $\mathrm{Cl}$ révèle une valeur en triméthylamine de 1,8\%, une telle teneur est considérée comme un signe d'altération du produit (Perombelon et al. , 1980 ; Kherrati et al. , 1998).

Les tests microbiologiques sont également favorables, puisque dans les essais C2, C3, C4 la disparition d'E. coli, des staphylocoques et des streptocoques a eu lieu au bout de 15 jours contrairement au témoin $\mathrm{Cl}$. Ainsi il y a eu une hygiénisation et une inhibition des protéolyses et des lipolyses dans les essais contenant de la levure brassicole en raison de son activité probiotique (Arneborg et al. , 1997). Ces tests montrent donc l'importance de la levure brassicole et confirment que son ajout aux déchets de poisson favorise une meilleure transformation et une inhibition des bactéries pathogènes et de dégradations. Ainsi le produit élaboré conserve ses qualités nutritives et demeure non toxique.

\section{CONCLUSION}

L'analyse physico-chimique des déchets de l'industrie halieutique montre leur teneur importante en phosphore et azote minéral. L'analyse microbiologique de ces déchets montre une charge microbienne importante, coliformes, staphylocoques, streptocoques. Une solution convenable et pratique s'impose. Cette étude montre qu'il est possible d'orienter la transformation de ces déchets pour produire facilement et à faible coût un produit utile, en les combinant à une fraction de déchets brassicoles riches en levures Saccharomyces cerevisiae, et ce grâce à un procédé très simple et maitrisé. Ce produit semble stable après 8 jours au vu de la stabilisation du $\mathrm{pH}$ pour les essais qui concorde avec les mesures de la conductivité.

Ce procédé permet une perte de matière limitée, et l'obtention d'un produit riche en phosphore et en azote minéral. II permet également une diminution des bactéries pathogènes et d'altérations lipolytiques (staphylocoques) et protéolytiques (streptocoques).

L'essai C2 possède ainsi de bonnes caractéristiques pour être utilisé en tant que fertilisant et a l'avantage d'exploiter une faible teneur de rejets brassicoles (uniquement 25\%) et le maximum en déchets de poissons. De plus l'application de ce fertilisant sur les cultures d'orge et de tomate présente de meilleurs résultats.

\section{Références bibliographiques}

AFILAL M.E., ELASRI O., MERZA Z. 20I4. Caractérisations des déchets organiques et évaluation du potentiel Biogaz (Organic waste characterization and evaluation of its potential biogas). J. Mater. Environ. Sci. №5 (4) pp : I I 60- I I 69.

AFNOR, NF V08-053, NF V08-057-I/2, LV 02-980I, 1993, 1994. Analyses à effectuer et principaux critères microbiologique pour l'homologation des matières fertilisantes et des supports de culture contenant des matières organiques d'origine animale ou végétale. Association française de normalisation, Paris. 
AFNOR, NFV I8-106, 1980. Aliments et produits animaux : Dosage du phosphore total, méthode spectrophotométrique. Association française de normalisation, Paris.

ALBRECHT R., 2007, Co-compostage de boues de station d'épuration et de déchets verts: nouvelles méthodologie du suivi des transformations de la matière organique. Thèse de doctorat, Université Paul Cezanne, Faculté des Sciences et Techniques, France.

ARNEBORG N., MOOS M.K., JAKOBSEN M., 1997. Induction of acetic acid tolerance and trehalose accumulation by added and produced ethanol in Saccharomyces cerevisiae. Biotecnol. Lett., n¹9(9), pp : 931-933.

BENZAKOUR M., SAISSI R., DIDIER G., 2003. Situation actuelle et évolution de la gestion des déchets solides au Maroc. Expérience pilote de la mise en place d'une décharge contrôlée à Essaouira. http://doc.abhatoo.net.ma/doc/ IMG/pdf/gestion_decharge.pdf

BRADSTREET R.B., 1965. The Kjeldahl Method for Organic Nitrogen. New York, NY: Academic Press Incorporated, pp : 39-88.

BRANGER A., RICHER M. M., ROUSTEL S., 2007. Microbiochimie et alimentation, Educagri Editions, $343 \mathrm{p}$.

CHABBAR AZ., 1996, Technologie de l'ensilage de poisson et disponibilité de la matière première au Maroc. Mémoire de 3ème cycle, IAV Hassan II, Rabat, Maroc.

EL MOUTAWAKIL A., 1995, Problèmes de la décharge de Kénitra. Actes du Séminaire sur le Compostage et la Gestion des Décharges, Kénitra, Maroc, pp : I03-। 12.

FAO, 2006. Utilisation des engrais par culture au Maroc Première édition, Rome, Italie.

JOUE (Journal Officiel de I'Union Européenne), 5 décembre 2005. RÈGLEMENT CE N²074/2005, L 338/27.

KHERRATI B., FAID M., EL YACHOUI M., WAHMANE A., 1998. Process for recycling slaughter houses wastes and by-products by fermentation. Bioresource Technology, N63( I), pp : 75-79.
LECLERCQ S., JACV.A., E.TINE, 1985, Essais de valorisation de sous-produits de brasserie par fermentation méthanique - procédé « contact aérobie ». Antenne ORSTROM de Microbiologie des Fermentations (UR 60 I) B* I 0047

LEYRAL G., VIERLING E., 2007. Microbiologie et toxicologie des aliments Hygiène et sécurité alimentaire. Biosciences et techniques: Sciences des Aliments, 4ème édition Reuil-Malmaison, CRDP d'Aquitaine, 290 p.

LOURHZAL W.,TAHRI E., FAID M., 2003. Ensilage des déchets de poisson et essai d'alimentation sur les rats. Revue Marocaine des Sciences Agronomiques et Vétérinaires, $n^{\circ} 23(1)$, pp : I5-20.

LOVERGROVEY., 1966. The determination of the dry weight of plankton and the effect of various factors on the values obtained. Sorne Contemporary Studies in Marine Science (Harold Bames Edo). George Allen and Unwin Ltd, London, pp : 429-467

MC CANCE R., 2002, The Composition of Foods. Widdowson's EM, Sixth Summary Edition, Cambridge, Food Standard Agency, 537 p.

MEYER A., DEIANA J., BERNARD A., 2004. Cours de microbiologie générale: avec problèmes et exercices corrigés, 2ème édition, Paris, Biosciences et techniques, $430 \mathrm{p}$.

PATRIE M. L., 2008 (Novembre), La levure de bière aliment nutritif et curatif. Membre du comité médiatique de l'Association des naturopathes agréés du Québec, Québec, Santé au Naturel. http://www.anaq.ca/articles/La\%20 levure\%20de\%20bi\%C3\%A8re.pdf

PEROMBELON M. C. M., KELMAN A., 1980. Ecology of the soft rot erwinias Annual Review of Phytopathology, n¹8, pp : 36I-387.

SHERSTHA K., SHERSTHA P., WALSH K. B., HARROWER K. M., MIDMORE D. J., 20 I I. Microbial enhancement of compost extracts based on cattle rumen content compost-Characterisation of a system. Bioresource Technology, $\mathrm{n}^{\circ}$ 102(17), pp : 8027-8034.

ZAHAR M., BENKERROUM N., GUEROUALI A., BAOU S., ALAHIANE L. 2002, Biological ensiling of sardine wastes in sugarcane molasse for their valorisation in animal feeding: microbiological study. Proceedings of International Symposium on Environmental Pollution Control and Waste Management, Tunis (EPCOWM'2002), Tunisie, pp : 304-311. 